\title{
Heavy OWA Operator of Trapezoidal Intuitionistic Fuzzy Numbers and Its Application to Multi-Attribute Decision Making
}

\author{
Chunlin LUO \\ School of Management, University of Chinese Academy Sciences, Beijing 100190, China; School of \\ Information Technology, Jiangxi University of Finance and Economics, Nanchang 330013, China \\ E-mail: chunlinluo@126.com \\ Xin TIAN* \\ Research Center on Fictitious Economy and Data Science, Chinese Academy of Sciences, Beijing \\ 100190, China; Key Research Laboratory of Big Data Mining and Knowledge Management, Chinese \\ Academy Sciences, Beijing 100190, China \\ E-mail: tianx@ucas.ac.cn \\ Shuping WAN \\ School of Information Technology, Jiangxi University of Finance and Economics, Nanchang 330013, \\ China \\ E-mail: shupingwan@163.com
}

\begin{abstract}
Heavy ordered weighted averaging (OWA) operator is important for characterizing the decision maker's attitudinal character in multi-attribute decision making (MADM) problem with part or total ignorance. This paper develops a new method based on heavy OWA operator to solve the MADM problem in which the attributes are characterized by some trapezoidal intuitionistic fuzzy numbers (TrIFNs). TrIFN, as a special kind of intuitionistic fuzzy set defined on the real numbers, is useful for characterizing the ill-known quantity in reality. Firstly, the operation laws and the cut sets concept for TrIFNs are introduced. Then the authors define the membership and non-membership average indexes. A new ranking method is developed on the basis of the two indexes. In the proposed decision model, the multi-attribute TrIFN values of the candidates are aggregated by the Heavy OWA operator, and ranked by their membership and non-membership average indexes. Lastly, the authors illustrate the proposed method by a numerical example which implies the practicality and effectiveness of the method.
\end{abstract}

Keywords Heavy OWA; trapezoidal intuitionistic fuzzy number; MADM; ranking

\section{Introduction}

Fuzzy sets is introduced by Zadeh ${ }^{[1]}$ to handle inexact and imprecise data. The core of the fuzzy set is that it provides a single membership value for each element without any hesitancy,

Received August 13, 2014, accepted September 11, 2014

*The corresponding author, E-mail: tianx@ucas.ac.cn

Supported by the National Natural Science Foundation of China (71461009, 71202114, 71261006) and Independent Innovation and Achievement Transformation Special Fund of Shandong Province (2014ZZCX03302) 
which implies that the evidence for $x \in X$ and the evidence against $x \in X$ are essentially equivalent. However, in reality, imprecise knowledge or information often results in vagueness and hesitancy. In order to tackle this problem, Atanassov ${ }^{[2]}$ proposed the intuitionistic fuzzy sets (IFS) by incorporating two characteristic functions characterizing the degree of membership and the degree of non-membership for each element respectively.

Fuzzy numbers, as a special case of fuzzy sets, are important to fuzzy multi-attribute decision making (MADM) problems. The core of the application is the ranking of the fuzzy numbers. Chu and Tsao ${ }^{[3]}$ developed a method for ranking fuzzy numbers with an area between the centroid point and original point. Abbasbandy and Hajjari ${ }^{[4]}$ proposed a new approach for ranking of trapezoidal fuzzy numbers based on the left and the right spreads at some $\sigma$-levels of trapezoidal fuzzy numbers.

As a generalization of the fuzzy number, an intuitionistic fuzzy number (IFN) seems to characterize an ill-known quantity more appropriately as discussed in [5]. Shu et al. ${ }^{[6]}$ defined the triangular IFN (TIFN) in a similar way to that of the fuzzy number ${ }^{[7]}$ and developed an algorithm for intuitionistic fuzzy fault tree analysis. $\mathrm{Li}^{[8]}$ defined the value index and ambiguity index for a TIFN and proposed a new method for ranking TIFNs based on a ratio of the value index to the ambiguity index.

Wang ${ }^{[9]}$ extended the concept of TIFN to the trapezoidal intuitionistic fuzzy number (TrIFN). Wang and Zhang ${ }^{[10]}$ explored the weighted averaging operators for TrIFNs and its applications to multi-criteria decision making problems. Wan and Dong ${ }^{[11]}$ defined the expectation and the expected score of TrIFNs from the geometric viewpoint and proposed the OWA and hybrid aggregation operators for TrIFNs. Wan ${ }^{[12]}$ considered the power average operators of TrIFNs.

In consideration of aggregating the information, the ordered weighted averaging (OWA) operator is a very common method. It was introduced by Yager in [13] and since its appearance it has been used in a wide range of application. One of its main characteristic is that it provides a parameterized family of aggregation operators that includes among others, the maximum, the minimum and the average criteria. Many researchers extended the OWA operator to the case with relation to fuzzy background. Wei ${ }^{[14]}$ proposed the trapezoidal fuzzy OWA operator and trapezoidal fuzzy hybrid aggregation operator. $\mathrm{Wu}$ and $\mathrm{Cao}^{[15]}$ proposed the intuitionistic trapezoidal fuzzy weighted geometric operator, ordered weighted geometric operator, the induced ordered weighted geometric operator and hybrid geometric operator.

The heavy OWA (HOWA) operator, proposed by Yager in [16], is an extension of the OWA operator. The motivation for using this operator is that when faced with part or total ignorance, the process of aggregating the payoffs will depend on the decision maker's attitudinal character. Therefore, the available information may not be aggregated by using usual averages bounded by the maximum and the minimum. For example, we may need the totaling type aggregation where the sum of the weights is $n$. The main characteristic of this type of operator is that it provides a wider class of aggregation operator by allowing the weighting vector to range between the OWA operator and the total operator. In other words, the difference with the OWA operators is that the sum of the weights is allowed to range between 1 and $n$ instead of being restricted to 1 . The heavy OWA provides a parameterized family of aggregation operators that includes among others, the minimum, the OWA operator and the total operator. The heavy OWA 
operator has also been extended by incorporating fuzzy measures (Yager ${ }^{[17]}$ ), by incorporating fuzzy numbers (Merigo and Casanovas ${ }^{[18]}$ ) and by incorporating distance measures (Merigo and Casanovas $\left.^{[19]}\right)$. More extension has also been studied by Merigo and Casanovas ${ }^{[20,21]}$. In this paper, we will extend the type of aggregation to the case where the attributes are characterized by TrIFNs.

\section{Trapezoidal Intuitionistic Fuzzy Numbers}

In this section, we first introduce the definition of TrIFNs, and then the operation laws for TrIFNs. Lastly, the cut sets and ranking order of TrIFNs are investigated.

Definition 1 (Wang and Zhang ${ }^{[9,10]}$ ) Let $\widetilde{a}$ be an intuitionistic fuzzy number in the set of real numbers, whose membership function and non-membership function are defined as follows:

$$
\mu_{\tilde{a}}(x)= \begin{cases}\frac{x-a}{b-a} w_{\widetilde{a}}, & \text { if } a \leq x<b \\ w_{\widetilde{a}}, & \text { if } b \leq x \leq c \\ \frac{d-x}{d-c} w_{\widetilde{a}}, & \text { if } c<x \leq d \\ 0, & \text { else }\end{cases}
$$

and

$$
\nu_{\tilde{a}}(x)= \begin{cases}\frac{b-x+\left(x-a_{1}\right) u_{\tilde{a}}}{b-a_{1}}, & \text { if } a_{1} \leq x<b, \\ \frac{u_{\tilde{a}},}{x-c+\left(d_{1}-x\right) u_{\tilde{a}}}, & \text { if } b \leq x \leq c, \\ d_{1}-c & \text { if } c<x \leq d_{1}, \\ 1, & \text { else, }\end{cases}
$$

respectively, where $a, b, c, d, a_{1}$ and $d_{1}$ are real numbers. The values $w_{\widetilde{a}}$ and $u_{\widetilde{a}}$ represent the maximum degree of membership and the minimum degree of non-membership, respectively, such that they satisfy the following conditions: $0 \leq w_{\tilde{a}} \leq 1,0 \leq u_{\tilde{a}} \leq 1, w_{\widetilde{a}}+u_{\tilde{a}} \leq 1$. Then the intuitionistic fuzzy number $\widetilde{a}$ is called a TrIFN, denoted by $\widetilde{a}=\left\langle\left([a, b, c, d] ; w_{\tilde{a}}\right),\left(\left[a_{1}, b, c, d_{1}\right] ; u_{\tilde{a}}\right)\right\rangle$.

If $[a, b, c, d]=\left[a_{1}, b, c, d_{1}\right]$, then the TrIFN $\widetilde{a}$ can be simply denoted by $\widetilde{a}=\left([a, b, c, d] ; w_{\tilde{a}}, u_{\tilde{a}}\right)$. In this paper, we hereafter refer to the TrIFNs in this form. The function $\pi_{\widetilde{a}}(x)=1-\mu_{\widetilde{a}}(x)-$ $\nu_{\tilde{a}}(x)$ denotes the hesitation of $\widetilde{a}$. The smaller $\pi_{\widetilde{a}}(x)$, the more certain $\widetilde{a}$. When $b=c$, a TrIFN reduces to a TIFN.

\subsection{Operation Laws for TrIFNs}

If $a \geq 0$ and $d>0$, then the $\operatorname{TrIFN} \widetilde{a}=\left([a, b, c, d] ; w_{\tilde{a}}, u_{\tilde{a}}\right)$ is called a positive $\operatorname{TrIFN}$ and denoted by $\tilde{a}>0$. The TrIFNs discussed in the following sections are all positive.

Definition 2 Let $\widetilde{a}_{1}=\left(\left[a_{1}, b_{1}, c_{1}, d_{1}\right] ; w_{\widetilde{a}_{1}}, u_{\widetilde{a}_{1}}\right), \widetilde{a}_{2}=\left(\left[a_{2}, b_{2}, c_{2}, d_{2}\right] ; w_{\widetilde{a}_{2}}, u_{\widetilde{a}_{2}}\right)$ be two TrIFNs and $\lambda \geq 0$. Then the operation laws for TrIFN are defined as follows:

1) $\widetilde{a}_{1}+\widetilde{a}_{2}=\left(\left[a_{1}+a_{2}, b_{1}+b_{2}, c_{1}+c_{2}, d_{1}+d_{2}\right] ; w_{\widetilde{a}_{1}} \wedge w_{\widetilde{a}_{2}}, u_{\widetilde{a}_{1}} \vee u_{\widetilde{a}_{2}}\right)$, where the symbols " $\wedge$ " and " $\vee$ " mean min and max operators, respectively.

2) $\widetilde{a}_{1} \widetilde{a}_{2}=\left(\left[a_{1} a_{2}, b_{1} b_{2}, c_{1} c_{2}, d_{1} d_{2}\right] ; w_{\widetilde{a}_{1}} \wedge w_{\widetilde{a}_{2}}, u_{\widetilde{a}_{1}} \vee u_{\widetilde{a}_{2}}\right)$;

3) $\lambda \widetilde{a}_{1}=\left(\left[\lambda a_{1}, \lambda b_{1}, \lambda c_{1}, \lambda d_{1}\right] ; w_{\widetilde{a}_{1}}, u_{\widetilde{a}_{1}}\right)$; 
4) $\widetilde{a}_{1}^{\lambda}=\left(\left[a_{1}^{\lambda}, b_{1}^{\lambda}, c_{1}^{\lambda}, d_{1}^{\lambda}\right] ; w_{\widetilde{a}_{1}}, u_{\widetilde{a}_{1}}\right)$.

It is easy to see that if $b_{i}=c_{i}(i=1,2)$, i.e., both $\widetilde{a}_{1}$ and $\widetilde{a}_{2}$ reduce to two TIFNs, then the operation laws of TrIFNs are degenerated to those of TIFN. Therefore, the operation laws of TrIFNs defined in this paper are the natural generalizations of that of TIFNs (see [8]).

According to Definition 2, the following properties naturally follows (see [8]):

1) $\widetilde{a}_{1}+\widetilde{a}_{2}=\widetilde{a}_{2}+\widetilde{a}_{1}, \widetilde{a}_{1} \widetilde{a}_{2}=\widetilde{a}_{2} \widetilde{a}_{1}$

2) $\lambda\left(\widetilde{a}_{1}+\widetilde{a}_{2}\right)=\lambda \widetilde{a}_{1}+\lambda \widetilde{a}_{2}, \lambda_{1} \widetilde{a}_{1}+\lambda_{2} \widetilde{a}_{1}=\left(\lambda_{1}+\lambda_{2}\right) \widetilde{a}_{1}, \widetilde{a}_{1}^{\lambda_{1}} \widetilde{a}_{1}^{\lambda_{2}}=\widetilde{a}_{1}^{\lambda_{1}+\lambda_{2}}$, where $\lambda \geq 0, \lambda_{1} \geq$ $0, \lambda_{2} \geq 0$

3) $\left(\widetilde{a}_{1}^{\lambda}\right)^{k}=\widetilde{a}_{1}^{\lambda_{1} k}$, where $\widetilde{a}_{1}>0$, and $\lambda \geq 0, k \geq 0$.

\subsection{Cut Sets and Ranking Order of TrIFNs}

Definition 3 A $\langle\alpha, \beta\rangle$-cut set of $\widetilde{a}=\left([a, b, c, d] ; w_{\tilde{a}}, u_{\tilde{a}}\right)$ is a crisp set of $R$, which is defined as

$$
\widetilde{a}_{\langle\alpha, \beta\rangle}=\left\{x \in R \mid \mu_{\widetilde{a}} \geq \alpha, v_{\widetilde{a}} \leq \beta\right\}
$$

where $0 \leq \alpha \leq w_{\tilde{a}}$; $0 \leq \beta \leq u_{\tilde{a}}$, and $0 \leq \alpha+\beta \leq 1$. Meanwhile, for any $\alpha \in\left[0, w_{\tilde{a}}\right]$ and $\beta \in\left[u_{\tilde{a}}, 1\right]$, an $\alpha$-cut set and $\beta$-cut set of $\widetilde{a}=\left([a, b, c, d] ; w_{\tilde{a}}, u_{\tilde{a}}\right)$ are defined respectively by

$$
\widetilde{a}_{\alpha}=\left\{x \in R \mid \mu_{\tilde{a}} \geq \alpha\right\}
$$

and

$$
\widetilde{a}^{\beta}=\left\{x \in R \mid v_{\widetilde{a}} \leq \beta\right\}
$$

The illustration of cut sets is shown as Figure 1.

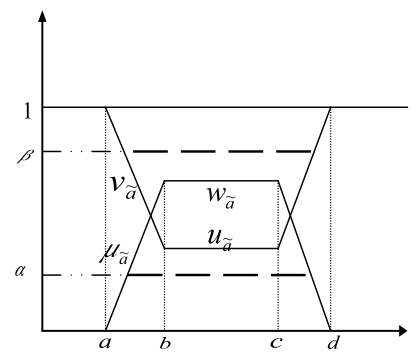

Figure 1 The illustration of cut sets

It is obvious that $\widetilde{a}_{\langle\alpha, \beta\rangle}=\widetilde{a}_{\alpha} \cap \widetilde{a}^{\beta}$.

Following the membership function of $\widetilde{a}$ and Definition 3 , it is easily seen that $\widetilde{a}_{\alpha}$ and $\widetilde{a}^{\beta}$ are closed interval and calculated respectively by

$$
\widetilde{a}_{\alpha}=\left[L_{\widetilde{a}}(\alpha), R_{\widetilde{a}}(\alpha)\right]=\left[\frac{\left(w_{\widetilde{a}}-\alpha\right) a+\alpha b}{w_{\widetilde{a}}}, \frac{\left(w_{\widetilde{a}}-\alpha\right) d+\alpha c}{w_{\widetilde{a}}}\right]
$$

and

$$
\widetilde{a}^{\beta}=\left[\widehat{L}_{\widetilde{a}}(\beta), \widehat{R}_{\widetilde{a}}(\beta)\right]=\left[\frac{(1-\beta) b+\left(\beta-u_{\tilde{a}}\right) a}{1-u_{\tilde{a}}}, \frac{(1-\beta) c+\left(\beta-u_{\tilde{a}}\right) d}{1-u_{\tilde{a}}}\right] .
$$

The ranking order of TrIFNs is a complicated problem which attracts many researchers. Nan et al. ${ }^{[22]}$ defined a ranking order of TIFNs based on the membership and nonmembership average index. We follow this idea and define the ranking order for TrIFNs as follows. 
Definition 4 Let $m\left(\widetilde{a}_{\alpha}\right)$ and $m\left(\widetilde{a}^{\beta}\right)$ be mean values of the intervals $\widetilde{a}_{\alpha}$ and $\widetilde{a}^{\beta}$ respectively, that is,

$$
m\left(\widetilde{a}_{\alpha}\right)=\frac{\left(w_{\widetilde{a}}-\alpha\right)(a+d)+\alpha(b+c)}{2 w_{\widetilde{a}}}
$$

and

$$
m\left(\widetilde{a}^{\beta}\right)=\frac{(1-\beta)(b+c)+\left(\beta-u_{\tilde{a}}\right)(a+d)}{2\left(1-u_{\tilde{a}}\right)} .
$$

Then the average index of the membership function $\mu_{\tilde{a}}(x)$ and the average index of the non-membership function $\nu_{\tilde{a}}(x)$ for the $\operatorname{TrIFN} \widetilde{a}$ are defined respectively by

$$
S_{\mu}(\widetilde{a})=\int_{0}^{w_{\tilde{a}}} m\left(\widetilde{a}_{\alpha}\right) \mathrm{d} \alpha=w_{\tilde{a}} \frac{a+b+c+d}{4}
$$

and

$$
S_{\nu}(\widetilde{a})=\int_{u_{\tilde{a}}}^{1} m\left(\widetilde{a}^{\beta}\right) \mathrm{d} \beta=\left(1-u_{\tilde{a}}\right) \frac{a+b+c+d}{4}
$$

Definition 5 Assume that $\widetilde{a}$ and $\widetilde{b}$ are two TrIFNs. $S_{\mu}(\widetilde{a})$ and $S_{\mu}(\widetilde{b})$ are the membership average indexes of $\widetilde{a}$ and $\widetilde{b}$, while $S_{v}(\widetilde{a})$ and $S_{v}(\widetilde{b})$ are the non-membership average indexes of $\widetilde{a}$ and $\widetilde{b}$ respectively. Then

(i) If $S_{\mu}(\widetilde{a})<S_{\mu}(\widetilde{b})$, then $\widetilde{a}$ is smaller than $\widetilde{b}$, and denoted by $\widetilde{a} \prec \widetilde{b}$ or $\widetilde{b} \succ \widetilde{a}$;

(ii) If $S_{\mu}(\widetilde{a})=S_{\mu}(\widetilde{b})$, then

(a) If $S_{v}(\widetilde{a})=S_{v}(\widetilde{b})$, then $\widetilde{a}$ and $\widetilde{b}$ represent the same amount and denoted by $\widetilde{a}=\widetilde{b}$;

(b) If $S_{v}(\widetilde{a})<S_{v}(\widetilde{b})$, then $\widetilde{a} \prec \widetilde{b}$.

Remark 6 The proposed ranking order for TrIFNs is reasonable and intuitive in consideration of the formulas (4) and (5) for the membership and non-membership average indexes. It means that the larger the $w_{\tilde{a}}$, the bigger the TrIFN; while the larger $u_{\tilde{a}}$, the smaller the TrIFN. The farther away from the origin the trapezoid of membership, the bigger the TrIFN.

\section{Heavy OWA Operators of TrIFNs}

Yager ${ }^{[16]}$ introduced an extension of the OWA operator called the heavy OWA operator which allows the weighting vector to range between the OWA operator and the total operator.

Definition 7 (Yager ${ }^{[16]}$ ) The heavy OWA aggregation operator of dimension $n$ is a mapping $H: R^{n} \rightarrow R$ such that $H\left(a_{1}, a_{2}, \cdots, a_{n}\right)=\sum_{j=1}^{n} w_{j} a_{\sigma(j)}$ where $a_{\sigma(j)}$ is the $j$ th largest of the arguments $\left\{a_{1}, a_{2}, \cdots, a_{n}\right\}$ and $w_{i}$ is the weight such that $0 \leq w_{i} \leq 1$ and $1 \leq \sum_{j=1}^{n} w_{j} \leq n$.

In the following, we generalize the heavy OWA operator to the TrIFN case and consider its applications in MADM problems.

Definition 8 Let $\Omega$ be the set of all TrIFNs. A heavy OWA operator of TrIFNs is a mapping $H: \Omega^{n} \rightarrow \Omega$ that has an associated weighting vector $W$ of dimension $n$ with $w_{j} \in[0,1]$ and $1 \leq \sum_{j=1}^{n} w_{j} \leq n$, such that $H\left(\widetilde{a}_{1}, \widetilde{a}_{2}, \cdots, \widetilde{a}_{m}\right)=\sum_{j=1}^{n} w_{j} \widetilde{a}_{\sigma(j)}$ where $\widetilde{a}_{\sigma(j)}$ is the $j$ th largest of the TrIFNs $\left\{\widetilde{a}_{1}, \widetilde{a}_{2}, \cdots, \widetilde{a}_{n}\right\}$.

According to Definitions 2 and 8 , we have: 
Theorem 9 Suppose that $\widetilde{a}_{i}=\left(\left[a_{i}^{(1)}, a_{i}^{(2)}, a_{i}^{(3)}, a_{i}^{(4)}\right] ; w_{\tilde{a}_{i}}, u_{\tilde{a}_{i}}\right)(i=1,2, \cdots, n)$ be a set of

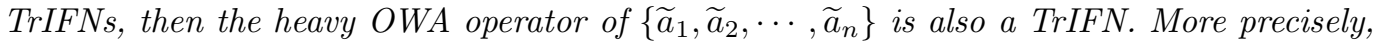

$$
\begin{aligned}
& H\left(\widetilde{a}_{1}, \widetilde{a}_{2}, \cdots, \widetilde{a}_{m}\right) \\
= & \sum_{j=1}^{n} w_{j} \widetilde{a}_{\sigma(j)} \\
= & \left(\left[\sum_{j=1}^{n} w_{j} a_{\sigma(j)}^{(1)}, \sum_{j=1}^{n} w_{j} a_{\sigma(j)}^{(2)}, \sum_{j=1}^{n} w_{j} a_{\sigma(j)}^{(3)}, \sum_{j=1}^{n} w_{j} a_{\sigma(j)}^{(4)}\right] ; \underset{1 \leq i \leq n}{\wedge} w_{\tilde{a}_{i}}, \underset{1 \leq i \leq n}{\vee} u_{\widetilde{a}_{i}}\right),
\end{aligned}
$$

where $\left\{w_{i}\right\}$ is the weight of the heavy OWA operator, and $\widetilde{a}_{\sigma(j)}=\left(\left[a_{\sigma(j)}^{(1)}, a_{\sigma(j)}^{(2)}, a_{\sigma(j)}^{(3)}, a_{\sigma(j)}^{(4)}\right], w_{\tilde{a}_{\sigma(j)}}\right.$, $\left.u_{\tilde{a}_{\sigma(j)}}\right)$ is the $j$ th largest of the TrIFNs $\left\{\widetilde{a}_{1}, \widetilde{a}_{2}, \cdots, \widetilde{a}_{n}\right\}$.

From Definition 8, we can easily find the following propositions.

Proposition 10 If $\left\{\widetilde{a}_{1}^{\prime}, \widetilde{a}_{2}^{\prime} \cdots, \widetilde{a}_{n}^{\prime}\right\}$ is any permutation of $\left\{\widetilde{a}_{1}, \widetilde{a}_{2}, \cdots, \widetilde{a}_{n}\right\}$, then

$$
H\left(\widetilde{a}_{1}^{\prime}, \widetilde{a}_{2}^{\prime} \cdots, \widetilde{a}_{n}^{\prime}\right)=H\left(\widetilde{a}_{1}, \widetilde{a}_{2}, \cdots, \widetilde{a}_{n}\right) .
$$

Proposition 11 (i) If $\sum_{j=1}^{n} w_{j}=n$, then heavy OWA operator of TrIFNs becomes the totaling operator;

(ii) If $w_{n}=1, w_{j}=0$ for all $j \neq n$, the heavy OWA operator of TrIFNs is degenerated to the minimum operator;

(iii) If $\sum_{j=1}^{n} w_{j}=1$, the heavy OWA operator of TrIFNs is reduced to the ordinary OWA operator; therefore, the heavy OWA operator of TrIFNs is a generalization of OWA operator of TrIFNs.

In the heavy OWA aggregation operator, an important quantity is the magnitude of the weighting vector $W$ which is defined by Yager ${ }^{[16]}$ as the sum of the elements of $W$ and denoted by $|W|$, namely, $|W|=\sum_{j=1}^{n} w_{j}$. It is obvious that:

Proposition $12 \quad H(\widetilde{a}, \widetilde{a} \cdots, \widetilde{a})=|W| \cdot \widetilde{a}$.

Proposition 12 directly shows the quantity $|W|$ characterizes the decision-maker's attitude.

In order to normalize the feature of the $W$, Yager introduced a characterizing parameter: The $\beta$ value of the vector $W$ which is defined as $\beta(W)=\frac{|W|-1}{n-1}$. It naturally follows that $\beta \in[0,1]$, as $|W| \in[1, n]$. Especially, for the pure totaling aggregation, we have $|W|=n$, and hence $\beta=1$; while for the ordinary OWA operator, we have $|W|=1$, and hence $\beta=0$. Moreover, if we define $\rho=1-\beta$, then $\rho$ characterizes the freedom or redundancy in the information being aggregated.

Based on the magnitude of the weighting vector $W$, we have

$$
\alpha(W)=\frac{1}{|W|} \sum_{j=1}^{n}\left(\frac{n-j}{n-1}\right) w_{j},
$$

which measures the attitudinal character of the weighting vector, and

$$
\operatorname{Dis}(W)=-\frac{1}{|W|} \sum_{j=1}^{n} w_{j} \ln \left(\frac{w_{j}}{|W|}\right),
$$

which denotes the entropy of dispersion of $W$. 


\section{MADM Model and Method Based on Heavy OWA Operators of TrIFNs}

In this section, we will apply the heavy OWA operators of TrIFNs to solve the corresponding MADM problem with relation to TrIFNs. Suppose that there exists an alternative set $A=$ $\left\{A_{1}, A_{2}, \cdots, A_{m}\right\}$, which represents $m$ non-inferior candidates from which the best one will be selected. Each candidate is assessed on $n$ attributes. Denote the attributes set by $X=$ $\left\{X_{1}, X_{2}, \cdots, X_{n}\right\}$. Assume that the assessment of the candidate $i$ over the attribute $j$ is characterized by a $\operatorname{TrIFN} \widetilde{a}_{i j}=\left(\left[h_{i j}^{(1)}, h_{i j}^{(2)}, h_{i j}^{(3)}, h_{i j}^{(4)}\right] ; \mu_{i j}, \nu_{i j}\right)$. In general, attributes can be classified into two types: Benefits attributes and cost attributes. In other words, the attributes set $F$ can be divided into two subsets: $F_{1}$ and $F_{2}$, which respectively represent the subsets of benefit attributes and cost attributes. Since the $n$ attributes may be measured in different ways, the matrix $\widetilde{A}=\left(\widetilde{a}_{i j}\right)_{m \times n}$ needs to be normalized into $\widetilde{R}=\left(\widetilde{r}_{i j}\right)_{m \times n}$ to eliminate the effect of different physical dimensions, where $\widetilde{r}_{i j}=\left(\left[r_{i j}^{(1)}, r_{i j}^{(2)}, r_{i j}^{(3)}, r_{i j}^{(4)}\right] ; \mu_{i j}, \nu_{i j}\right)$. In this paper, the normalization method is chosen as follows:

$$
\begin{aligned}
& r_{i j}^{(k)}=\frac{h_{i j}^{(k)}-\min _{i} h_{i j}^{(1)}}{\max _{i} h_{i j}^{(4)}-\min _{i} h_{i j}^{(1)}}, \quad \text { for } j \in F_{1} \\
& r_{i j}^{(k)}=\frac{\max _{i} h_{i j}^{(4)}-h_{i j}^{(5-k)}}{\max _{i} h_{i j}^{(4)}-\min _{i} h_{i j}^{(1)}}, \quad \text { for } j \in F_{2}
\end{aligned}
$$

where $i=1,2, \cdots, m, j=1,2, \cdots, n, k=1,2,3,4$.

The normalization method above is to guarantee that the elements of a normalized trapezoidal fuzzy number $\left[r_{i j}^{(1)}, r_{i j}^{(2)}, r_{i j}^{(3)}, r_{i j}^{(4)}\right]$ lie in the unit interval $[0,1]$. Therefore, the decision matrix $\widetilde{A}=\left(\widetilde{a}_{i j}\right)_{m \times n}$ can be transformed into the normalized trapezoidal intuitionistic fuzzy decision matrix $\widetilde{R}=\left(\widetilde{r}_{i j}\right)_{m \times n}$.

To reflect the decision maker's attitudinal character appropriately, we allow the sum of the weights to range between 1 and $n$ instead of being restricted to 1 . And the attributes of the candidates are aggregated by this heavy OWA operator of TrIFNs. The candidates are ranked by their aggregation values. Thus, an algorithm and process of the MADM problems using TrIFN can be summarized as follows:

Step 1 Normalize the TrIFN decision matrix. According to Equation (6) or (7), the original decision matrix $\widetilde{A}=\left(\widetilde{a}_{i j}\right)_{m \times n}$ can be normalized to $\widetilde{R}=\left(\widetilde{r}_{i j}\right)_{m \times n}$.

Step 2 Rearrange the TrIFNs $\left\{\widetilde{r}_{i 1}, \widetilde{r}_{i 2}, \cdots, \widetilde{r}_{i n}\right\}$ for each candidate $A_{i}$. Calculate the membership and non-membership average indexes for $\widetilde{r}_{i 1}, \widetilde{r}_{i 2}, \cdots, \widetilde{r}_{i n}$ and reorder the TrIFNs for each candidate $A_{i}$ according to Definition 5 .

Step 3 Use the heavy OWA operator of TrIFNs to aggregate $\widetilde{r}_{i 1}, \widetilde{r}_{i 2}, \cdots, \widetilde{r}_{i n}$ for each candidate $A_{i}$. Suppose the weighting vector is $W=\left(w_{1}, w_{2}, \cdots, w_{n}\right)$, then the collective overall TrIFN of candidate $A_{i}$ is obtained as

$$
H\left(A_{i}\right)=\left(\left[\sum_{j=1}^{n} w_{j} r_{i \sigma(j)}^{(1)}, \sum_{j=1}^{n} w_{j} r_{i \sigma(j)}^{(2)}, \sum_{j=1}^{n} w_{j} r_{i \sigma(j)}^{(3)}, \sum_{j=1}^{n} w_{j} r_{i \sigma(j)}^{(4)}\right] ; \min _{1 \leq j \leq n}\left\{\mu_{i j}\right\}, \max _{1 \leq j \leq n}\left\{\nu_{i j}\right\}\right)
$$


where $\widetilde{r}_{i \sigma(j)}$ represents the $j$ th largest of the TrIFNs $\left\{\widetilde{r}_{i 1}, \widetilde{r}_{i 2}, \cdots, \widetilde{r}_{i n}\right\}$.

Step 4 Rank the candidates. For each candidate, calculate the membership and nonmembership average indexes for its collective overall TrIFN $H\left(A_{i}\right)$, then the ranking orders of candidates are generated according to the ranking method of TrIFNs in Definition 5.

\section{Numerical Analysis}

\subsection{An Application in the Personnel Selection Problem}

We illustrate the proposed ranking method by a personnel selection problem. Suppose that a company wants to hire a department manager, and there are three candidates (denoted by $\left.A_{1}, A_{2}, A_{3}\right)$ applying for the position. The decision making committee assesses the three candidates through five attributes, including emotional steadiness $\left(X_{1}\right)$, oral communication skill $\left(X_{2}\right)$, personality $\left(X_{3}\right)$, past experience $\left(X_{4}\right)$ and self-confidence $\left(X_{5}\right)$. The scores of the candidates with respect to the five attributes are given in Table 1.

Table 1 Decision matrix given by committee

\begin{tabular}{|c|c|c|c|c|}
\hline$X_{1}$ & $X_{2}$ & $X_{3}$ & $X_{4}$ & $X_{5}$ \\
\hline$A_{1}([5,7,8,9] ; 0.7,0.1)$ & $([2,3,4,5] ; 0.8,0.2$ & $([3,4,6,7] ; 0.5,0.2)$ & $([2,4,6,7] ; 0.6,0.3)$ & ;0.6, 0.3) \\
\hline$A_{2}([3,4,5,6] ; 0.9,0.1)$ & $([3,4,5,8] ; 0.5,0.4)$ & $([4,6,7,9] ; 0.6,0.4)$ & $([4,5,6,8] ; 0.7,0.2)$ & $([5,7,8,9] ; 0.4,0.4)$ \\
\hline$A_{3}([5,6,7,8] ; 0.8,0.1)$ & $([1,3,5,8] ; 0.6,0.2)$ & $([1,3,6,7] ; 0.7,0.2)$ & $([2,4,5,7] ; 0.6,0.4)$ & $([2,3,4,6] ; 0.8,0.2)$ \\
\hline
\end{tabular}

The five attribute are benefit attributes. According to (6), the normalized TrIFN decision matrix is obtained as in Table 2.

Table 2 Normalized decision matrix

\begin{tabular}{lll}
\hline \multicolumn{1}{c}{$X_{1}$} & $X_{2}$ & $X_{3}$ \\
\hline$A_{1}([0.33,0.67,0.83,1.00] ; 0.7,0.1)$ & $([0.14,0.29,0.43,0.57] ; 0.8,0.2)$ & $([0.25,0.38,0.63,0.75] ; 0.5,0.2)$ \\
$A_{2}([0.00,0.17,0.33,0.50] ; 0.9,0.1)$ & $([0.29,0.43,0.57,1.00] ; 0.5,0.4)$ & $([0.38,0.63,0.75,1.00] ; 0.6,0.4)$ \\
$A_{3}([0.33,0.50,0.67,0.83] ; 0.8,0.1)$ & $([0.00,0.29,0.57,1.00] ; 0.6,0.2)$ & $([0.00,0.25,0.63,0.75] ; 0.7,0.2)$ \\
\hline \hline$X_{4}$ & $X_{5}$ \\
\hline$A_{1}([0.00,0.33,0.67,0.83] ; 0.6,0.3)$ & $([0.00,0.25,0.63,0.88] ; 0.6,0.3)$ \\
$A_{2}([0.33,0.50,0.67,1.00] ; 0.7,0.2)$ & $([0.50,0.75,0.88,1.00] ; 0.4,0.4)$ \\
$A_{3}([0.00,0.33,0.50,0.83] ; 0.6,0.4)$ & $([0.13,0.25,0.38,0.63] ; 0.8,0.2)$ \\
\hline
\end{tabular}

Table 3 Membership and non-membership average indexes matrix

\begin{tabular}{llllll}
\hline & $X_{1}$ & $X_{2}$ & $X_{3}$ & $X_{4}$ & $X_{5}$ \\
\hline$A_{1}$ & $\langle 0.50,0.64\rangle$ & $\langle 0.29,0.29\rangle$ & $\langle 0.25,0.40\rangle$ & $\langle 0.27,0.32\rangle$ & $\langle 0.26,0.31\rangle$ \\
$A_{2}$ & $\langle 0.23,0.23\rangle$ & $\langle 0.29,0.34\rangle$ & $\langle 0.41,0.41\rangle$ & $\langle 0.44,0.50\rangle$ & $\langle 0.31,0.47\rangle$ \\
$A_{3}$ & $\langle 0.47,0.52\rangle$ & $\langle 0.28,0.37\rangle$ & $\langle 0.29,0.33\rangle$ & $\langle 0.25,0.25\rangle$ & $\langle 0.28,0.28\rangle$ \\
\hline
\end{tabular}

To reorder the normalized TrIFNs for each candidate, we need to calculate the membership average index $S_{\mu}$ and non-membership average index $S_{v}$ for each normalized TrIFN. We denote 
the pair of membership and non-membership average indexes by $\left\langle S_{\mu}, S_{v}\right\rangle$ and calculate the pair for each normalized TrIFN as shown in Table 3.

Suppose the initial weighting vector is $W_{0}=(0.1,0.2,0.2,0.2,0.3)$. But, after careful analysis, the committee believe it underestimates the candidates for part ignorance of the candidates. The reason may be that the committee neglect the potential of the candidates over the attributes. So the committee decide to increase the weighting vectors by $50 \%$ to reflect its attitudinal character. Thus, the final weighting vector is $W=(0.15,0.3,0.3,0.3,0.45)$. Using the above information of ranking order of the normalized TrIFNs of $A_{1}$ over the attributes, we can calculate the heavy ordered weighted value for the candidate $A_{1}$ as follows:

$$
\begin{aligned}
H\left(A_{1}\right)= & 0.15 *([0.33,0.67,0.83,1.00] ; 0.7,0.1)+0.3 *([0.14,0.29,0.43,0.57] ; 0.8,0.2) \\
& +0.3 *([0.00,0.33,0.67,0.83] ; 0.6,0.3)+0.3 *([0.00,0.25,0.63,0.88] ; 0.6,0.3) \\
& +0.45 *([0.25,0.38,0.63,0.75] ; 0.5,0.2) \\
= & ([0.20,0.53,0.93,1.17] ; 0.5,0.3) .
\end{aligned}
$$

Applying the same method, we can obtain the collective overall TrIFNs of candidate $A_{2}$ and $A_{3}$ as

$$
\begin{aligned}
H\left(A_{2}\right)= & 0.15 *([0.33,0.50,0.67,1.00] ; 0.7,0.2)+0.3 *([0.38,0.63,0.75,1.00] ; 0.6,0.4) \\
& +0.3 *([0.50,0.75,0.88,1.00] ; 0.4,0.4)+0.3 *([0.29,0.43,0.57,1.00] ; 0.5,0.4) \\
& +0.45 *([0.00,0.17,0.33,0.50] ; 0.9,0.1) \\
= & ([0.40,0.69,0.91,1.28] ; 0.4,0,4)
\end{aligned}
$$

and

$$
\begin{aligned}
H\left(A_{3}\right)= & 0.15 *([0.33,0.50,0.67,0.83] ; 0.8,0.1)+0.3 *([0.00,0.25,0.63,0.75] ; 0.7,0.2) \\
& +0.3 *([0.00,0.29,0.57,1.00] ; 0.6,0.2)+0.3 *([0.13,0.25,0.38,0.63] ; 0.8,0.2) \\
& +0.45 *([0.00,0.33,0.50,0.83] ; 0.6,0.4) \\
= & ([0.09,0.46,0.80,1.21] ; 0.6,0.4) .
\end{aligned}
$$

Using the formulas (4) and (5), we can calculate the membership and non-membership average indexes of $H\left(A_{i}\right)$ as follows:

$$
\begin{aligned}
& S_{\mu}\left[H\left(A_{1}\right)\right]=0.354 \text { and } S_{v}\left[H\left(A_{1}\right)\right]=0.495, \\
& S_{\mu}\left[H\left(A_{2}\right)\right]=0.328 \text { and } S_{v}\left[H\left(A_{2}\right)\right]=0.492, \\
& S_{\mu}\left[H\left(A_{3}\right)\right]=0.384 \text { and } S_{v}\left[H\left(A_{3}\right)\right]=0.384 .
\end{aligned}
$$

Thus, the ranking order of the three candidates is obtained as $A_{3} \succ A_{1} \succ A_{2}$ according to Definition 5. So the best candidate is $A_{3}$.

\subsection{Comparison with Extant Heavy OWA MADM Method}

In the above illustrated example, if we use the interval numbers to characterize the committee's evaluation, then Table 1 can be rewritten as Table 4 through deleting the corresponding membership and non-membership indexes. 
Table 4 New decision matrix given by committee

\begin{tabular}{llllll}
\hline & $X_{1}$ & $X_{2}$ & $X_{3}$ & $X_{4}$ & $X_{5}$ \\
\hline$A_{1}$ & {$[5,7,8,9]$} & {$[2,3,4,5]$} & {$[3,4,6,7]$} & {$[2,4,6,7]$} & {$[1,3,6,8]$} \\
$A_{2}$ & {$[3,4,5,6]$} & {$[3,4,5,8]$} & {$[4,6,7,9]$} & {$[4,5,6,8]$} & {$[5,7,8,9]$} \\
$A_{3}$ & {$[5,6,7,8]$} & {$[1,3,5,8]$} & {$[1,3,6,7]$} & {$[2,4,5,7]$} & {$[2,3,4,6]$} \\
\hline
\end{tabular}

Merigo and Casanovas ${ }^{[21]}$ utilized the heavy OWA operator to MADM with uncertain information. To further explain the importance of using TrIFNs to characterize the information of assessment, we apply the method proposed in [21] to solve the corresponding personnel selection problem. After computation, the uncertain heavy OWA values of alternatives are obtained as follows:

$$
\begin{aligned}
\operatorname{UHOWA}\left(A_{1}\right) & =[3.45,5.70,8.40,10.20], \\
\operatorname{UHOWA}\left(A_{2}\right) & =[5.70,7.65,9.15,11.55], \\
\operatorname{UHOWA}\left(A_{3}\right) & =[2.85,5.25,7.65,10.50] .
\end{aligned}
$$

According to the criteria for ranking interval numbers as shown in [21], we find that $A_{2} \succ$ $A_{1} \succ A_{3}$. So the best candidate is $A_{2}$.

It is worth noting that the ultimate ranking orders obtained by this paper and by Merigo and Casanovas ${ }^{[21]}$ are very different. The main reason is that the membership and non-membership indexes are lost in the interval numbers, which weakens the ability of characterizing the assessment information. As stated in Introduction, TrIFNs are defined by incorporating the membership and non-membership function, which makes the membership degrees and nonmembership degrees no longer relative to a fuzzy concept "Excellent" or "Good", but relative to the trapezoidal fuzzy number. Thus, the information given by decision makers can be reflected more exactly. Hence, TrIFNs may better characterize the assessment information of decision problems than the interval numbers by adding the membership and non-membership indexes. So, the ranking order obtained in this paper seems more reasonable than that obtained by $[21]$.

\section{Conclusions}

In this paper, we generalized the heavy OWA operator, which is important for characterizing the decision maker's attitudinal character in MADM problem with ignorance, to the TrIFNs and applied the method to solve the MADM problem in which the attributes are characterized by TrIFNs. TrIFN, a special type of intuitionistic fuzzy set, is of importance for characterizing the ill-known quantity in our life. After introducing the operation laws and the cut sets concept for TrIFNs, we defined the membership and non-membership average indexes for TrIFNs. Then we proposed a new decision method where the multi-attribute TrIFN values of the candidate are aggregated by the Heavy OWA operator and ranked by the membership and non-membership average indexes. At last, we illustrated the proposed method through a numerical example which shows the practicality and effectiveness of the method.

Due to the fact that the TrIFN is a generalization of a trapezoidal fuzzy number, the other 
existing method of ranking fuzzy numbers may be extended to TrIFN. More effective ranking method of TrIFN will be investigated in the near future.

\section{References}

[1] Zadeh L A. Fuzzy sets. Information and Control, 1965, 8(3): 338-353.

[2] Atanassov K T. Intuitionistic fuzzy sets. Fuzzy Sets and Systems, 1986, 20(1): 87-96.

[3] Chu T, Tsao C. Ranking fuzzy numbers with an area between the centroid point and original point. Computers and Mathematical with Applications, 2002, 43: 111-117.

[4] Abbasbandy S, Hajjari S. A new approach for ranking of trapezoidal fuzzy number. Computers and Mathematics with Applications, 2009, 57(3): 413-419.

[5] Li D F. A note on using intuitionistic fuzzy sets for fault-tree analysis on printed circuit board assembly. Microelectronics Reliability, 2008, 48: 1741.

[6] Shu M H, Cheng C H, Chang J R. Using intuitionistic fuzzy sets for fault tree analysis on printed circuit board assembly. Microelectronics Reliability, 2006, 46(12): 2139-2148.

[7] Dubois D, Prade H. Fuzzy Sets and Systems: Theory and Applicaion. Academic Press, New York, 1980.

[8] Li D F. A ratio ranking method of triangular intuitionistic fuzzy numbers and its application to MADM problems. Computers and Mathematics with Applications, 2010, 60: 1557-1570.

[9] Wang J Q. Overview on fuzzy multi-creteria decision-making approach. Control and Decision, 2008, 23(6): 601-607.

[10] Wang J Q, Zhang Z. Aggregation operators on intuitionistic trapezoidal fuzzy numbers and its application to multi-criteria decision making problem. Journal of Systems Engeneering and Electronics, 2009, 20(2): 321-326.

[11] Wan S P, Dong J Y. Method of trapezoidal intuitionistic fuzzy number for multi-attribute group decision. Control and Decision, 2010, 25(5): 773-776.

[12] Wan S P. Power average operators of trapezoidal intuitionistic fuzzy numbers and application to multiattribute group decision making. Applied Mathematics Modelling, 2013, 37(6): 4112-4126.

[13] Yager R R. On ordered weighted averaging aggregation opertors in multi-criteria decision making. IEEE Transactions on Systems, Man and Cybernatics, 1988, 18: 183-190.

[14] Wei G W. Some arithmetic aggregation operators with intuitionistic trapezoidal fuzzy numbers and their application to group decision making. Journal of Computers, 2010, 5(3): 345-351.

[15] Wu J, Cao Q W. Same families of geometric aggregation operators with intuitionistic trapezoidal fuzzy numbers. Applied Mathematical Modelling, 2013, 37: 318-327.

[16] Yager R R. Heavy OWA operators. Fuzzy Optimization and Decision Making, 2002, 1(4): 379-397.

[17] Yager R R. Monitored heavy fuzzy measures and their role in decision making under uncertainty. Fuzzy Sets and Systems, 2003, 139(3): 491-513.

[18] Merigo J M, Casanovas M. Using fuzzy numbers in heavy aggregation operators. International Journal of Information and Communication Engineering, 2008, 4(7): 487-492.

[19] Merigo J M, Casanovas M. Induced and heavy aggregation operators with distance measures. Journal of Systems Engineering and Electronics, 2010, 21(3): 431-439.

[20] Merigo J M. New extensions to the OWA operators and their application in decision making. Department of Business Administration, University of Barcelona, 2008.

[21] Merigo J M, Casanovas M. Induced and uncertain heavy OWA operator. Computers and Industrial Engineering, 2011, 60: 106-116.

[22] Nan J X, Li D F, Zhang M J. A lexicographic method for matrix games with payoffs of triangular intuitionistic fuzzy numbers. International Journal of Computational Intelligence Systems, 2010, 3(3): 280-289. 